\title{
Introducing deprescribing into culture of medication
}

A

n Ontario pharmacist has received a government grant to develop clinical guidelines to help doctors determine whether patients are on medications they no longer need or that should be reduced.

"We don't normally test drugs in the elderly, but they are taking many drugs. As they get older and get more chronic conditions, the number of medications increases," says Barbara Farrell, a clinical scientist with the Bruyère Research Institute in Ottawa, Ontario.

Sometimes when medications are deprescribed or reduced, "confusion will clear, or they'll stop falling, and a lot of literature supports that," says Farrell, who received the $\$ 430000$ grant from the Ontario Ministry of Health and Long-Term Care.

Over the next three years, her team will develop, implement and evaluate the guidelines one at a time, at both primary and long-term care sites. One of the main goals is to change the way people think about pharmacy among the elderly. Farrell hopes the guidelines will prompt routine re-evaluation of drugs or sets of drugs and provoke thought about how long a patient needs a drug and how dosage should change with age.

"It's introducing an approach, deprescribing, into the culture of how we think about medication," she says.

Taking multiple drugs simultaneously, or polypharmacy, is common among the elderly. Nearly two-thirds of seniors were on five or more drugs in 2008 - and $21 \%$ took at least $10-$ according to a report by the Canadian Institute for Health Information (CIHI).

More drugs means more potential for adverse drug events. According to a 2013 CIHI study, $13 \%$ of seniors taking five or more prescription medications experienced adverse drug events that required medical attention, compared to $6 \%$ for those taking only one or two drugs.

In her work, Farrell has seen that assessing the benefits and risks of a

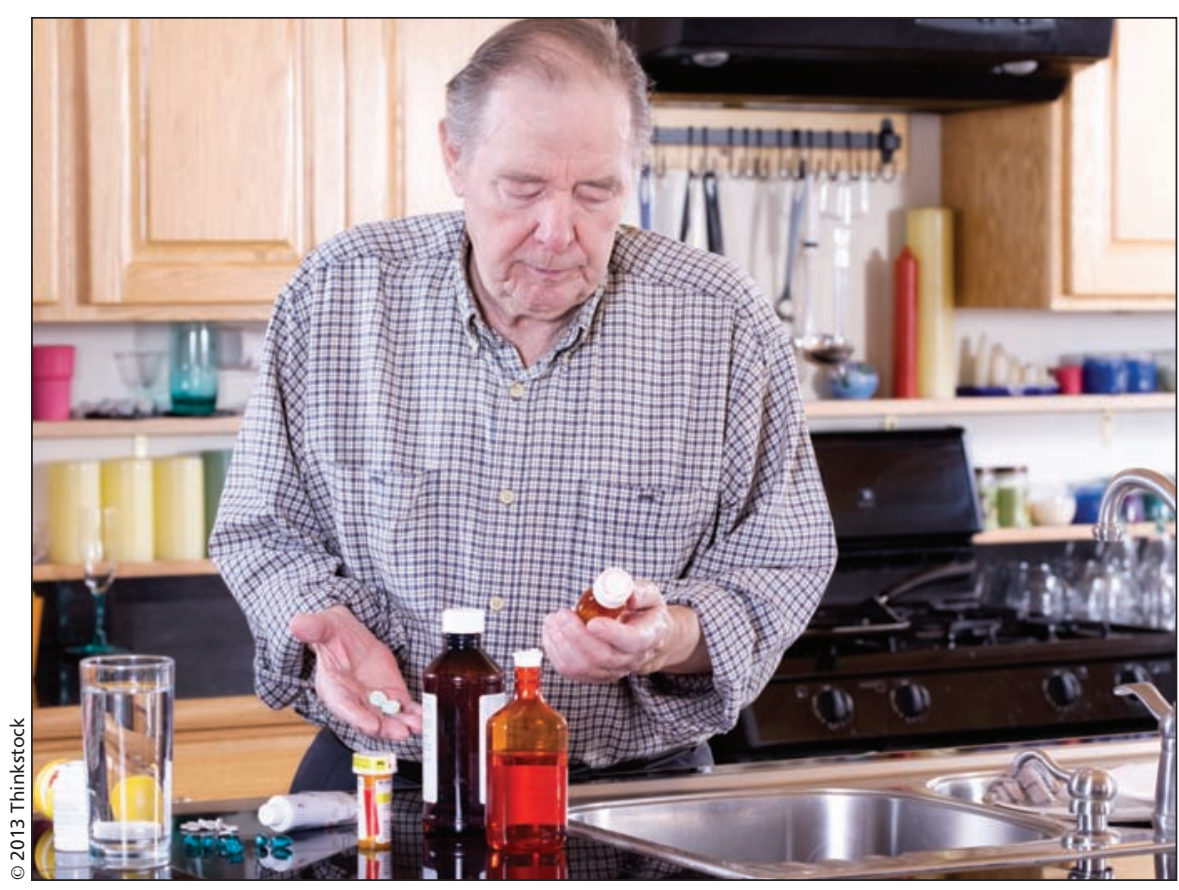

Assessing the benefits and risks of a medication, followed by tapering off or discontinuing certain drugs, can improve health in elderly patients

medication, followed by tapering off or discontinuing certain drugs, has caused marked improvements in elderly patients. Still, it can be difficult for physicians to know which medications to stop, how to stop or taper them and what to monitor during the process.

Many elderly patients see multiple specialists, and information about the duration and use of a drug does not always reach the primary physician, often leaving doctors "hesitant to stop something someone else started," Farrell says.

Another challenge with creating guidelines for deprescribing is that older patients present "a unique set of variables," says Dr. Jan Schuling, a general practitioner trainer who researches deprescription at University Medical Center Groningen in the Netherlands. He emphasizes the need for shared decision-making between experts and patients.

"People, especially older people, are individuals, each with their own values.
Only the patient himself can tell us, what he thinks important in life," he wrote in an email.

Doctors also tend, in some cases, to be uncomfortable discussing deprescribing with patients, Schuling and colleagues found in a 2012 study (BMC Fam Pract 2012;13:56). Schuling's team conducted group interviews with Dutch doctors, discovering that they were sometimes hesitant to broach topics they felt would make their patients uncomfortable, including discussions of life expectancy and scaling back preventative medication.

"[T]hey are concerned that patients may interpret this as a sign of being given up on," states the study.

Dr. Schuling recommends, above all, that any deprescription guidelines emphasize a patient-centred approach, focusing on a patient's preferences and the treatment burden that they may experience. - Catherine Cross, CMAJ

CMAJ 2013. DOI:10.1503/cmaj.109-4554 